\title{
Supplementary information to
}

\section{Optically manipulated microtools to measure adhesion of the nanoparticle targeting ligand glutathione to brain endothelial cells}

Tamás Fekete ${ }^{1,2, \#, ~ M a ́ r i a ~ M e ́ s z a ́ r o s ~}{ }^{1, \#, ~ Z s o l t ~ S z e g l e t e s ~}{ }^{1}$, Gaszton Vizsnyiczai ${ }^{1}$, László Zimányi ${ }^{1}$, Mária A. Deli ${ }^{1}$, Szilvia Veszelka ${ }^{1 *}$, Lóránd Kelemen ${ }^{*}$

1) Institute of Biophysics, Biological Research Centre, Eötvös Loránd Research Network (ELKH), 6726 Szeged, Hungary

2) Doctoral School in Multidisciplinary Medicine, University of Szeged, 6720 Szeged, Hungary

\# These authors contributed equally to this manuscript.

*kelemen.lorand@brc.hu and szilvia.veszelka@brc.hu 


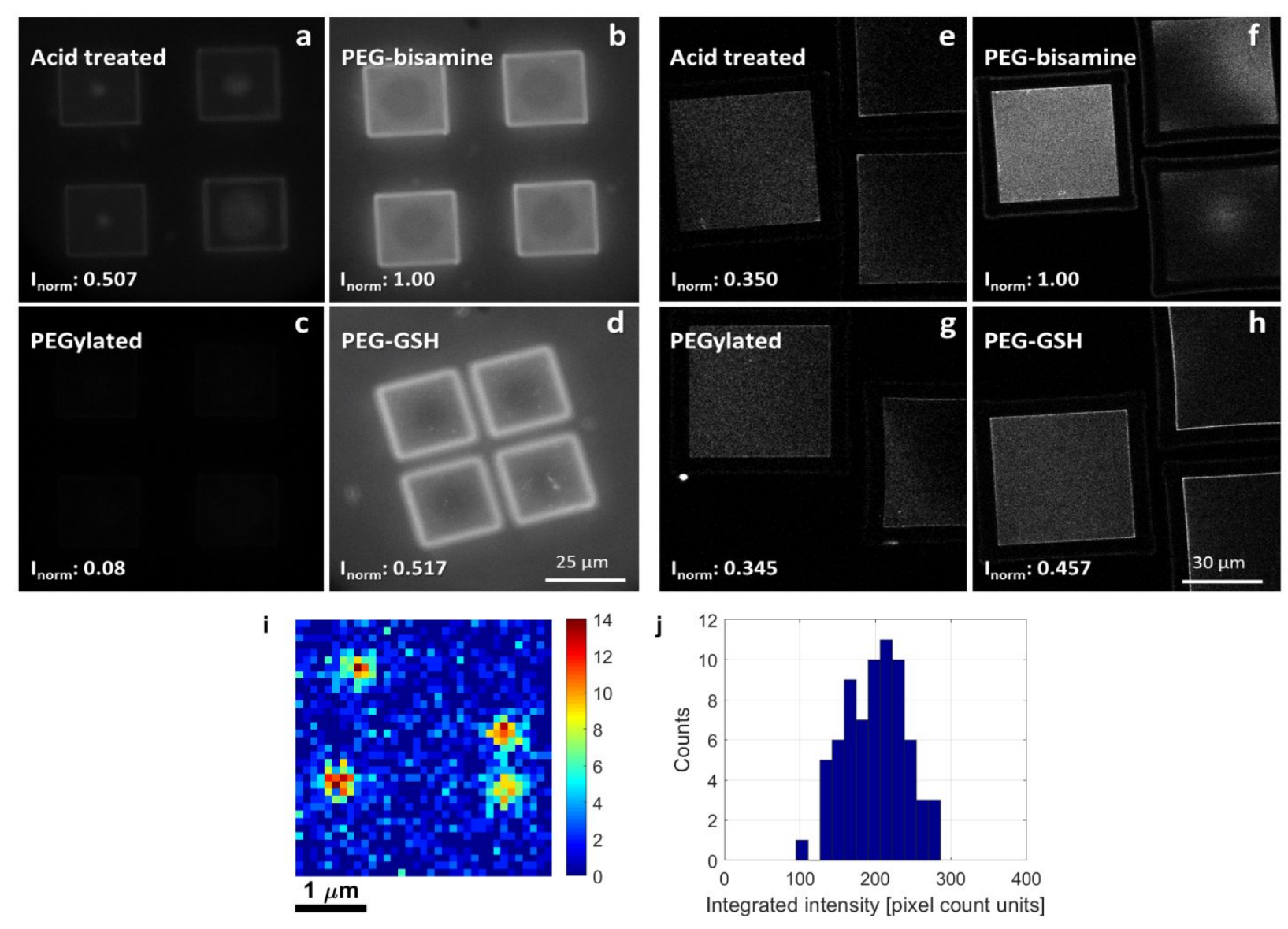

Figure S1. Fluorescence microscopy images of differently functionalized SU8 blocks; the blocks were fluorescently labelled with CY-5-NHS-ester dye. Wide-field fluorescence microscopy (a-d) as well as laser scanning confocal fluorescence microscopy $(\boldsymbol{e}-\boldsymbol{h})$ were used for the evaluation. The blocks, functionalized with the homobifunctional PEG-bisamine (b, $\boldsymbol{f}$ ), served as positive control. After this treatment only primary amine groups coat the surface and it produces the maximum surface density of this group achievable with our protocol. The acid-treated surfaces $(\boldsymbol{a}, \boldsymbol{e})$ served as one of the negative controls, where no PEG linker was used at all; to these, the fluorescent dye can bind only aspecifically. $\mathrm{NH}_{2}-\mathrm{PEG}$-maleimide (PEGylated) treated surfaces $(\boldsymbol{c}, \boldsymbol{g})$ served as the other negative control. Since the $\mathrm{NH}_{2}$ group of this linker reacts with the acid-treated SU8 surface, the maleimide groups at the other end of the PEG chain are presented towards the fluorescent stain, which can only bind to it aspecifically due to the lack of amine groups. The GSH functionalized (PEG-GSH) blocks $(\boldsymbol{d}, \boldsymbol{h})$ always show higher fluorescence intensity than the negative controls, supporting that GSH is indeed immobilized covalently on top of SU-8 with the PEG-maleimide linker. On all wide-field images the average background intensity was subtracted from the intensity measured in the very center of the blocks, and then these intensity values were normalized with that of the positive control resulting the normalized $\boldsymbol{I}_{\text {norm }}$ intensity values. The confocal images show only the frame of the top layer of the fluorescent block, so the normalization was done without the correction with the background. The scalebar on $\boldsymbol{d}$ is applied to a-d and that on $\boldsymbol{h}$ to $\boldsymbol{e}$-h. The surface coverage of GSH was determined with amine-reactive CY5 dye. The integrated intensity determined from the fluorescent images of single CY5 molecules (i) were used to calculate the surface coverage of CY5 on the homogeneously coated layers (the colorbar shows fluorescent intensity in pixel count units). The intensity of the single fluorophores was quite uniform, giving a well-defined average integrated intensity value (j) $(N=71)$. 

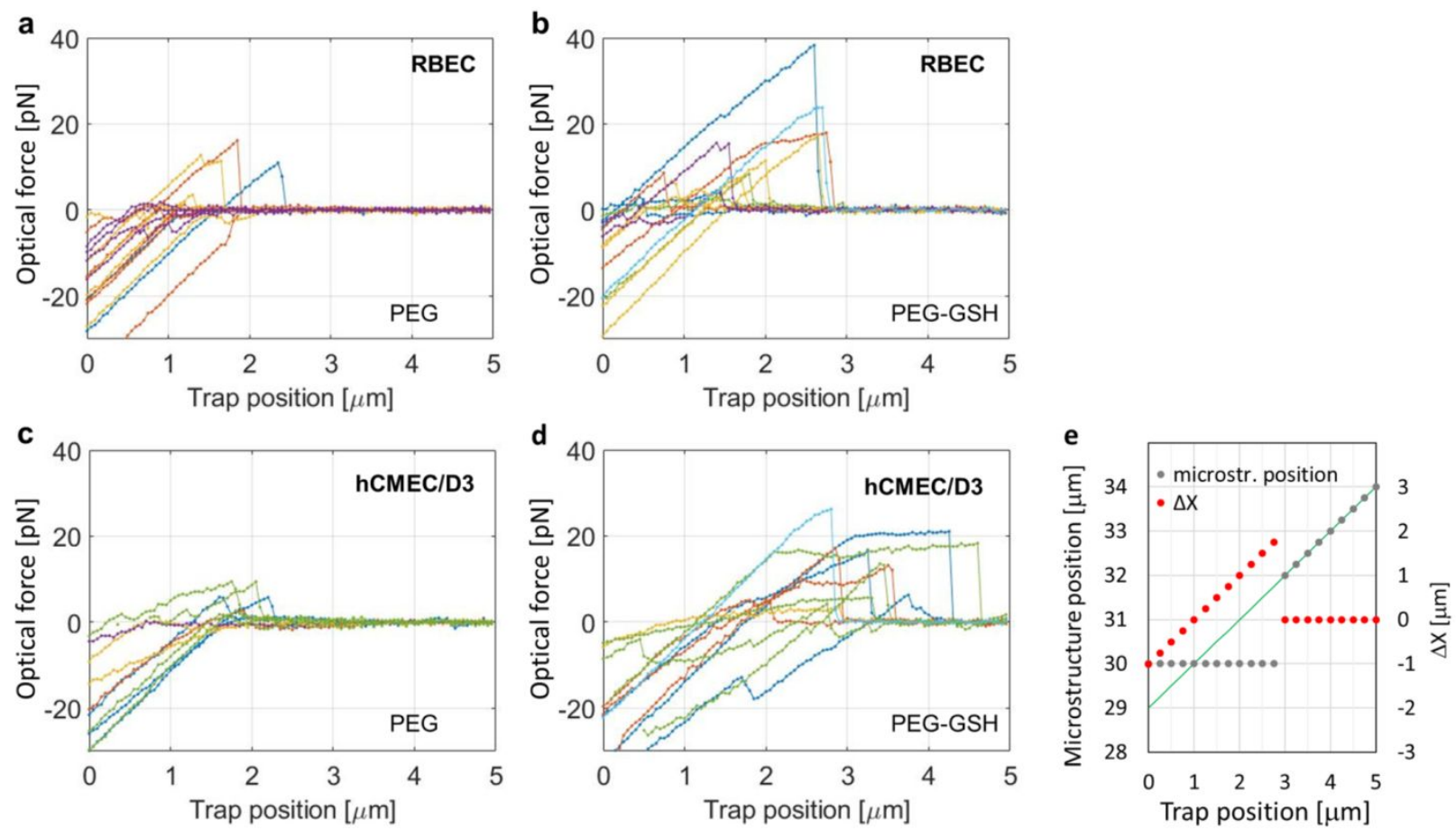

Figure S2. Optical force curves from all experiments on rat brain endothelial cells (RBEC) (a, b) and on human cerebral microvascular endothelial cells (hCMEC/D3) $(\boldsymbol{c}, \boldsymbol{d})$ measured with $50 \mathrm{~nm}$ step size using PEGylated (PEG) $(\boldsymbol{a}, \boldsymbol{c})$ and GSH functionalized (PEG-GSH) $(\boldsymbol{b}, \boldsymbol{d})$ microtools. In each graph, traces of the same color were obtained with the same microtool but on different cells. (e) Illustrates the calculation of $\Delta X$ from the microtool position as the function of the trapping focus position ( 0 refers to the start of the pulling of the microtool). Grey circles show modelled microtool positions along the direction of the pulling in the image coordinate system. In this example, in the 0-3 $\mu \mathrm{m}$ trap position range the microtool does not move due to its adhesion to the cell. At $3 \mu \mathrm{m}$ it separates from the cell and above $3 \mu \mathrm{m}$ it precisely follows the trapping beam. The grey line is the fit to this last, 3-5 $\mu \mathrm{m}$ section. $\Delta X$ is calculated by subtracting the microtool positions from the fitted line. The optical forces are then simply the product of $\Delta X$ and the trap stiffness. 

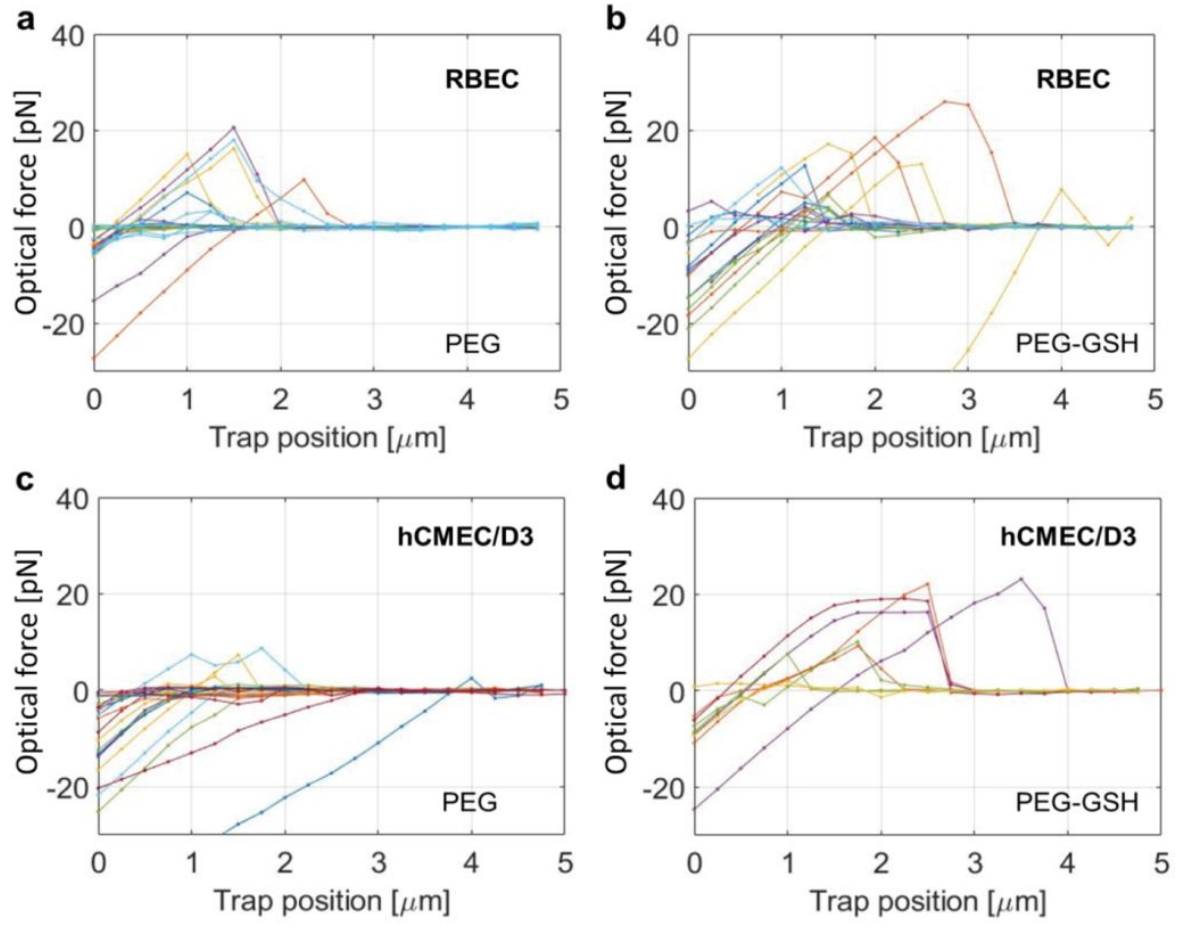

Figure S3. Optical force curves from all experiments on rat brain endothelial cells (RBEC) (a, b) and on human cerebral microvascular endothelial cells $(h C M E C / D 3)(c, d)$ measured with $250 \mathrm{~nm}$ step size using PEGylated (PEG) $(\boldsymbol{a}, \boldsymbol{c})$ and GSH functionalized (PEG-GSH) $(\boldsymbol{b}, \boldsymbol{d})$ microtools. In each graph traces of the same color were obtained with the same microtool but on different cells. 


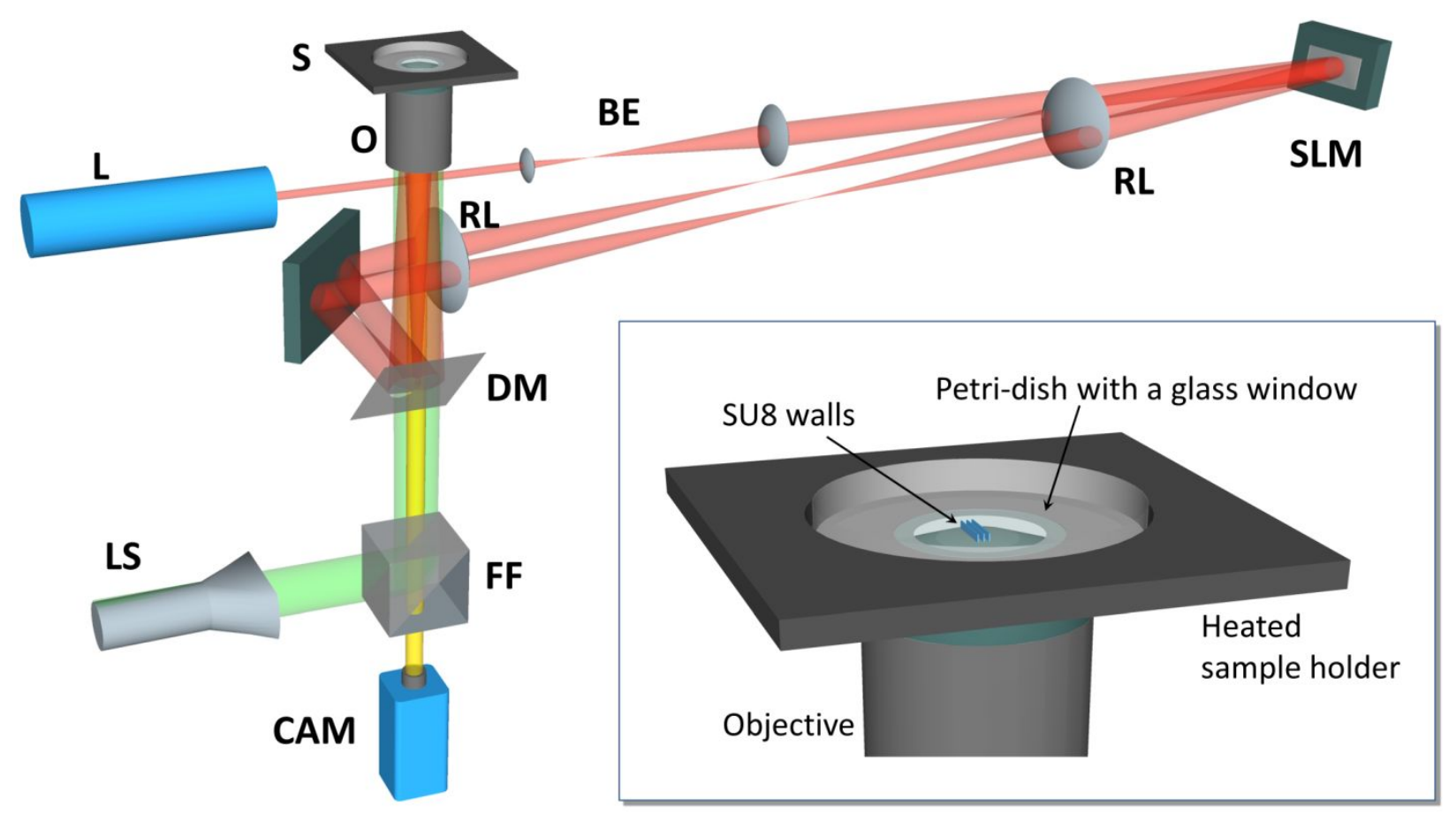

Figure S4. Optical manipulation setup. The key elements are the following: laser source for trapping $(L)$, beam expander (BE), spatial light modulator (SLM), relay lenses (RL1 and RL2), dichroic mirror (DM), fluorescence filter set (FF), light source (LS), camera (CAM), microscope objective (O), sample (S). The laser beam is shown doubled after the SLM to visualize the capabilities of the holographic optical tweezers (HOT) that can split the original beam into several beams and can also steer them independently. The insert shows the close-up of the heated sample holder that is situated over the objective and nests the Petri-dish with the glass bottom. This glass supports the vertical SU8 walls on which the cells were cultured (not to scale). 